\title{
A back door to the neuron
}

An electrochemical gene-delivery method enables rapid modification of gene expression in postmitotic neurons in vivo, changing their identity and connectivity pattern.

It is a Kafkaesque era for biology: under the right 'genetic spell', cells of a certain identity can wake up one morning metamorphosed into another. But there are still unanswered questions about the boundaries of this cellular plasticity.

Can, for example, one type of neuron be engineered into another in the living brain? Can a set of genes change a cell's characteristic morphology and firing pattern, and even rewire it into a different network?

Although it is known that manipulating gene expression in early neuronal progenitor cells can modify the identity of their progeny, it is not known whether neurons can be reprogrammed in this manner after the animal's birth.

A major hurdle in investigating these questions has been the inability to rapidly and efficiently manipulate gene expression in specific postmitotic neurons. Classical
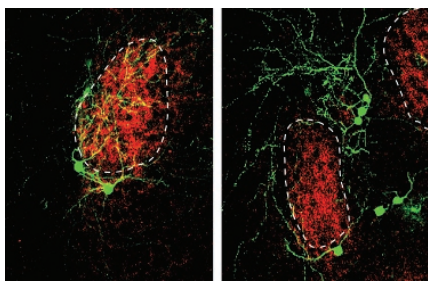

Changing the wiring pattern of L4 neurons (green cells, left) by ectopic Fezf2 expression (right). Reprinted from Nature Neuroscience. electroporation works efficiently only in mitotic cells, and infection with viral vectors takes days for the transgene to be expressed. Undeterred, Denis Jabaudon, at the University of Geneva in Switzerland, and his team set out to answer these questions and to find the right tools to do so.

Jabaudon and his group wanted to change the identity of layer 4 (L4) neurons of the mouse brain, which receive input connections from the thalamus, and to find out whether doing so altered the cells' characteristic connectivity pattern.

The team first worked to optimize the protocols for electroporation of DNA plasmids into the brain of postnatal animals. But this alone didn't do the trick. "It had always puzzled me

\section{GENOMICS}

\section{SINGLE CELLS GO FULLY GENOMIC}

A semilinear amplification method allows uniform amplification across a single cell's genome.

Sunney Xie from Harvard University has long been interested in the study of single molecules. Starting with work on single-molecule enzymology, which he says "got me a job here at Harvard," he has recently focused on single-cell genomics. For Xie, measuring the sequence of the 46 chromosomes in a human cell is just another form of single-molecule measurement. Single molecule to the extreme, if you will.

Gathering his postdoctoral fellow Chenghang Zong and graduate student Sijia Lu, Xie initially advised them to use multiple displacement amplification (MDA) to amplify the genetic content of single cells. In MDA, developed by Roger Lasken in 2001, random primers anneal to target DNA and are amplified by a high-fidelity polymerase at ambient temperature. MDA represented the current state of the art and had been successfully used for genome amplification from single cells. But the team ran into problems: the highest genome coverage they obtained was $60 \%$, and amplification bias prevented them from achieving even coverage across all of the genome. "The fundamental problem," says Xie, "is exponential amplification; it magnifies the difference."

So the team went back to the drawing board to replace exponential with linear amplification. They developed MALBAC (multiple annealing and looping-based amplification cycles) which involves annealing of random tagged primers to sheared genomic DNA, followed by extension with a strand-replacement polymerase. The resulting semiamplicons contain a tag at the 3 ' end, and, after another cycle, the complementary tag at the 5 ' end, resulting in the looping of the amplicon. This loop no longer serves as a template for primer hybridization and thus is not further amplified. At the end of five cycles, the pool of looped amplicons is subjected to PCR to produce the micrograms of DNA needed for high-throughput sequencing.

In direct comparison to MDA, MALBAC shows great promise. The team achieved genomic coverage of $93 \%$, which allowed them to detect copy-number variations (CNVs) in single 
why electroporation didn't work in postmitotic neurons in an efficient and reproducible way," says Jabaudon. "Then I stumbled across several reports that suggested that plasmids get stuck in the cytoplasm and never enter the nucleus." So he and his team searched the literature for compounds that could help enhance the nuclear transport of the plasmids. They settled on a compound, TCHD (trans-cyclohexane-1,2-diol), which acts as a nuclear permeabilizing agent.

Injection of a volume of TCHD-solubilized DNA plasmid into the mouse brain and delivery of a specific electroporation protocol caused a reporter gene to be expressed in the targeted cortical region in just a few hours. They named the procedure 'iontoporation'.

Iontoporation worked well only during the first postnatal week, but if gene expression at later stages was desired, the group could inject tamoxifen-inducible plasmids early on and activate gene expression in adulthood.

The team used this method to ectopically express a transcription factor specific to layer $5 \mathrm{~B}$ neurons, Fezf2, in L4 neurons 1 day after birth, and they examined the outcome a couple of weeks later. Fezf2 was an ideal candidate to act as an identity switch, as it is both necessary and sufficient to generate L5B neurons during development.

They examined the molecular signature of the targeted L4 cells, their morphology and their firing properties, and it was clear to them that the cells had become L5B neurons. Even the short- and long-range connectivity inputs to the engineered cells now resembled those of L5B neurons. Cell-type conversion, however, worked only if the ectopic gene was expressed during the animal's first 10 days of life. After that, L4 neurons were L4 neurons for good.

It will now be interesting to study whether these neurons can be switched into other neuron varieties and whether identity switching can happen in other types of neurons. To investigate these possibilities, the group has started to test the protocol's efficacy in other brain areas.

\section{Erika Pastrana}

\section{RESEARCH PAPERS}

De la Rossa, A. et al. In vivo reprogramming of circuit connectivity in postmitotic neocortical neurons. Nat. Neurosci. 16, 193-200 (2013).

cancer cells (Zong et al., 2012). Alec Chapman, a graduate student in the Xie lab, working together with Zong, provided the statistical analyses to prove that, in Xie's words, "it is beyond doubt that MALBAC has higher coverage and precision."

Yet Xie is worried that "when other people try it, they will get frustrated and think the method does not work" because unbiased amplification is only one of the challenges when it comes to single-cell analysis. Another important issue is the risk of contamination. To minimize contamination, Zong and Lu used a clean room for their experiments, but Xie also recommends the use of microfluidics.

The design of the random primers for MALBAC also requires careful thought. Researchers who do not want to put in the time to design the primers themselves may soon have access to a commercial kit distributed by Yikon Genomics, a start-up company Lu is involved with.

There are many applications for which single-cell analysis is important. Understanding the genetic variation in tumors is one of them. Xie's team showed that they could measure the mutation rate in a cancer cell line by comparing single-nucleotide polymorphisms between a founder cell and daughter cells 20 generations later. Their measured rate was 2.5 bases in the whole genome per cell division.

Analysis of single meiotic cells is another application. In a companion paper (Lu et al., 2012), the Harvard team, collaborating with scientists at the Biodynamic Optical Imaging Center of Peking University, used MALBAC followed by high-throughput sequencing on sperm cells. The data allowed them to phase $80 \%$ of the donor's heterozygous single-nucleotide polymorphisms. With the resulting haplotypes, they could draw a map of crossover sites in each sperm, with a resolution of at least 200 kilobases for each recombination event. Xie predicts that these maps will be very helpful in better understanding male infertility.

Nicole Rusk

\section{RESEARCH PAPERS}

Lu, S. et al. Probing meiotic recombination and aneuploidy of single sperm cells by whole-genome sequencing. Science 338, 1627-1630 (2012).

Zong, C. et al. Genome-wide detection of single-nucleotide and copy-number variations of a single human cell. Science 338, 1622-1626 (2012). 